\title{
AN ANN BASED BRAIN ABNORMALITY DETECTION USING MR IMAGES
}

\author{
Prof. K. V. Kulhalli ${ }^{1}$ and Prof. S. R. Khot ${ }^{2}$ \\ ${ }^{1,2}$ Associate professor, D. Y. Patil College of Engineering and Technology, \\ Kolhapur, Maharashtra. \\ kvkulhalli@gmail.com, srkhot08@gmail.com
}

\begin{abstract}
The Main purpose of this paper is to design, implement and evaluate a strong automatic diagnostic system that increases the accuracy of tumor diagnosis in brain using MR images. This presented work classifies the brain tissues as normal or abnormal automatically, using computer vision. This saves lot of radiologist time to carryout monotonous repeated job. The acquired MR images are processed using image preprocessing techniques. The preprocessed images are then segmented, and the various features are extracted. The extracted features are fed to the artificial neural network as input that trains the network using error back propagation algorithm for correct decision making.
\end{abstract}

\section{KEYWORDS}

Magnetic Resonance Imaging, Computer Tomography, Artificial Neural Network , Histogram Equalization, Brain Abnormality.

\section{INTRODUCTION}

Brain Cancer is a disease that causes abnormal cell division and spreads over different parts of body through blood and lymphatic system. Tumors are divided into two categories as benign and malignant tumor. The major problem in the medical field is the early diagnosis of the disease .This may be due to many reasons such as the lack of specialization and infrastructural facility.

The commonly used imaging techniques are Computer Tomography (CT) scan, Sonography and Magnetic Resonance Imaging (MRI) technique. As MRI technique differentiates clearly the soft tissues, has high resolution, and produces good contrast hence is being adopted. Early and automatic Brain Tumor Detection increases the survival rate of the patients and thus is a need of the present era.

The presented system acquires the MR images of the patient with the complaint, Acquired images are resized and are converted in to grey scale images. The Preprocessing operation such as filtering is carried out for noise elimination and enhancing the image quality for better contrast. From the preprocessed image the Intensity and Texture based features are extracted. The extracted features are further used as input to the Artificial Neural Network (ANN) and the network is trained in such a way to classify the tumor as malignant or benign. 


\section{SYSTEM DESIGN AND IMPLEMENTATION}

\subsection{Block schematic of the diagnostic system}

The block schematic of the ANN based Brain Tumor diagnostic system to be implemented is as shown in Fig.1.

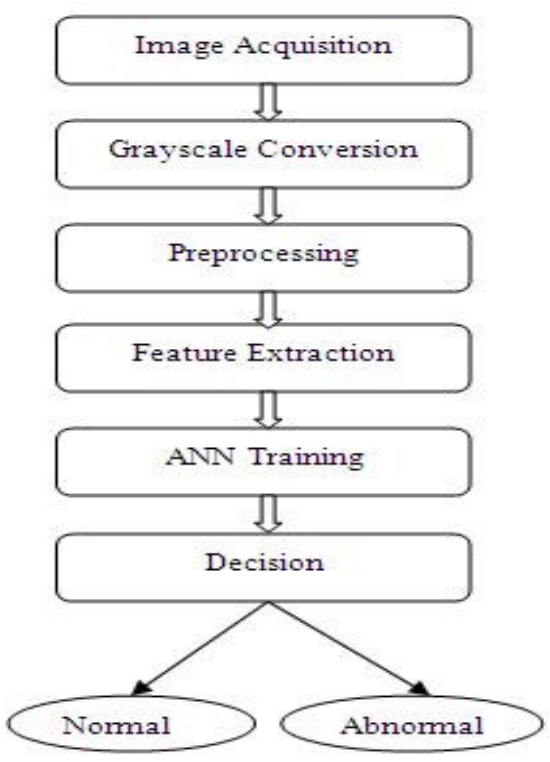

Fig1 Block Diagram of the diagnostic system

The designed system is implemented and tested using MATLAB. The GUI of different stages is shown in the following snapshots.

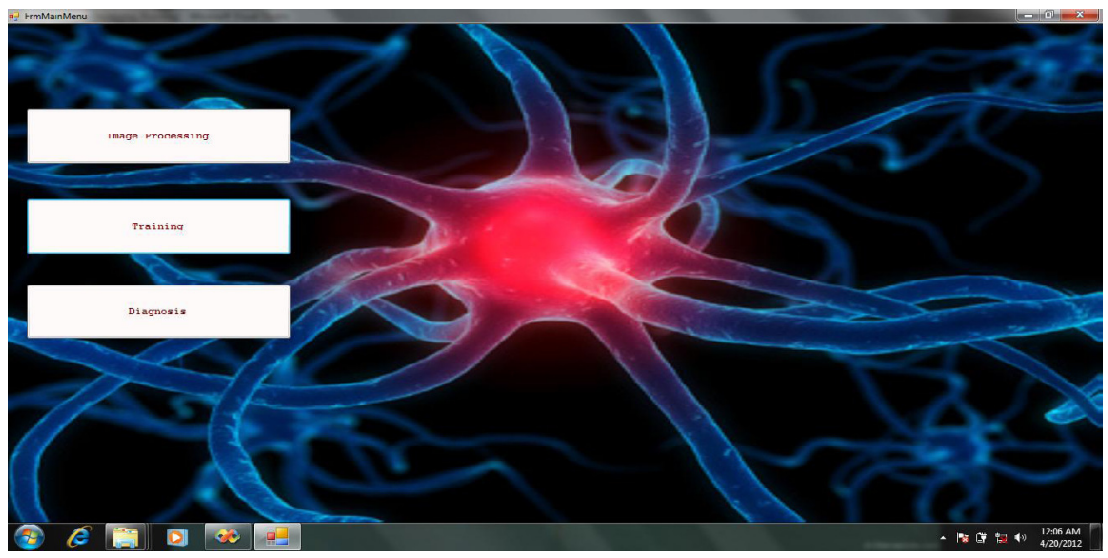

Fig 2. GUI of the total system presented 


\subsection{Image acquisition}

MR images for the patients who suffer from brain complaints are acquired from the hospitals, oncologists, radiologists and medical atlases.

\subsection{Gray Scale Conversions}

Acquired images are then resized and converted into Grayscale image in which the value of each pixel is a single sample, i.e. it carries only intensity information. These images have many shades of gray in between 0 to 255 . They are also called monochromatic, denoting the presence of only one (mono) color (chrome). They are fast to process and need less storage space.

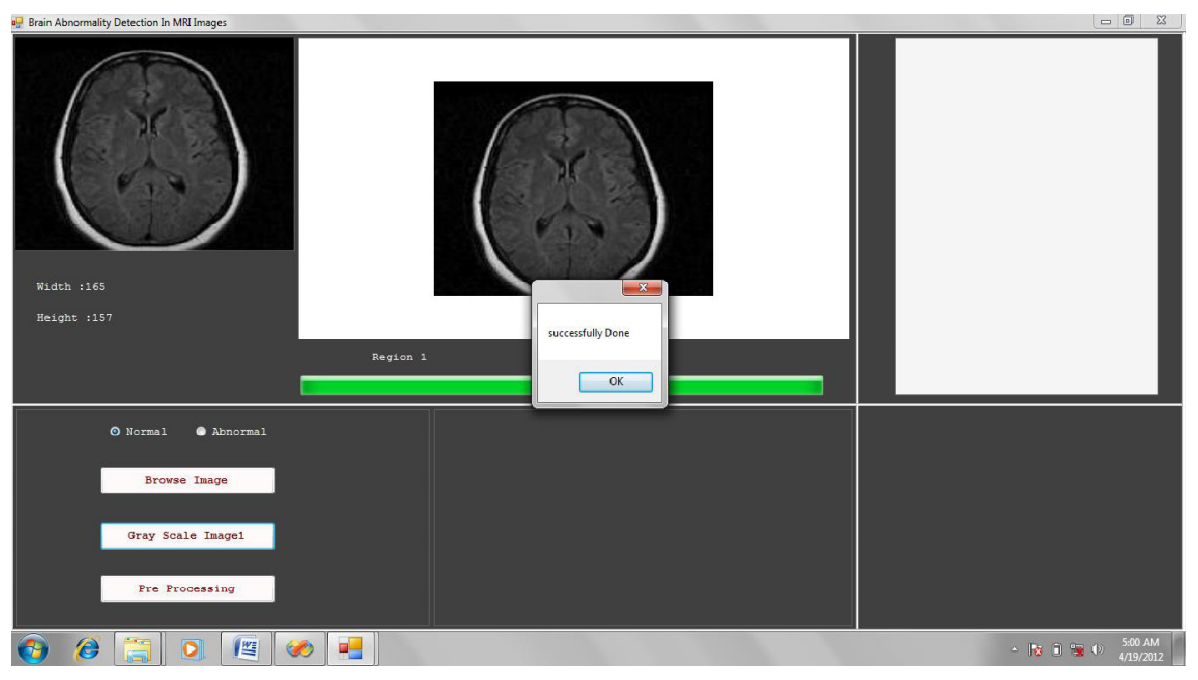

Fig 3. GUI of Gray scale conversion of selected MR image.

\subsection{Image Preprocessing}

The pre-processing improves the image data and suppresses undesired distortions thus enhances some image features which are relevant for further processing and analysis. In this paper, pixel brightness transformations and local pre-processing methods are realized using Mat lab.

Histogram Equalization (HE) increases the global contrast of the images, especially when the data of the image is represented by its close contrast values. Through this adjustment, the intensities are better distributed on the histogram. This allows for areas of lower local contrast to gain a higher contrast. HE accomplishes this by effectively spreading out most frequent intensity values.

The median filter which is a nonlinear digital filter, used to remove noise, to smoothen and to preserve the edges of the image, because edges are of critical importance for the visual appearance of images. The Sobel operator is used for edge detection as it is superior compared with other gradient operators used. It also reduces the noise by inherent averaging of neighboring pixels. 


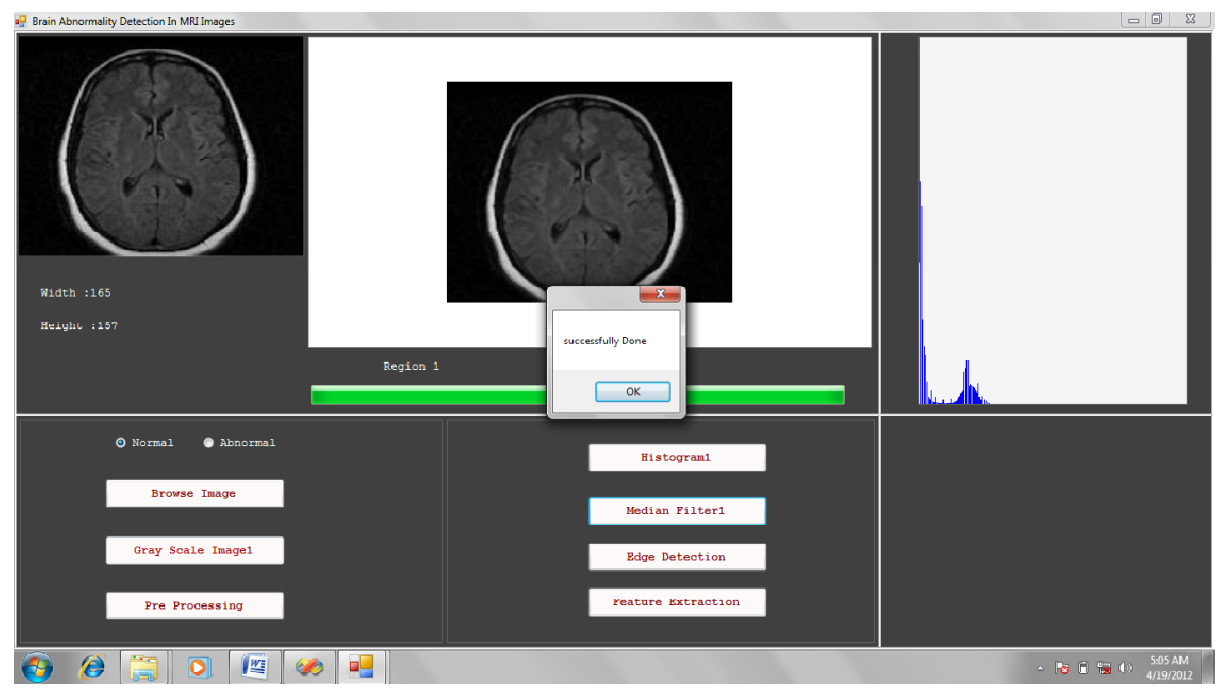

Fig 4. GUI of the Median filtered image of selected MR image.

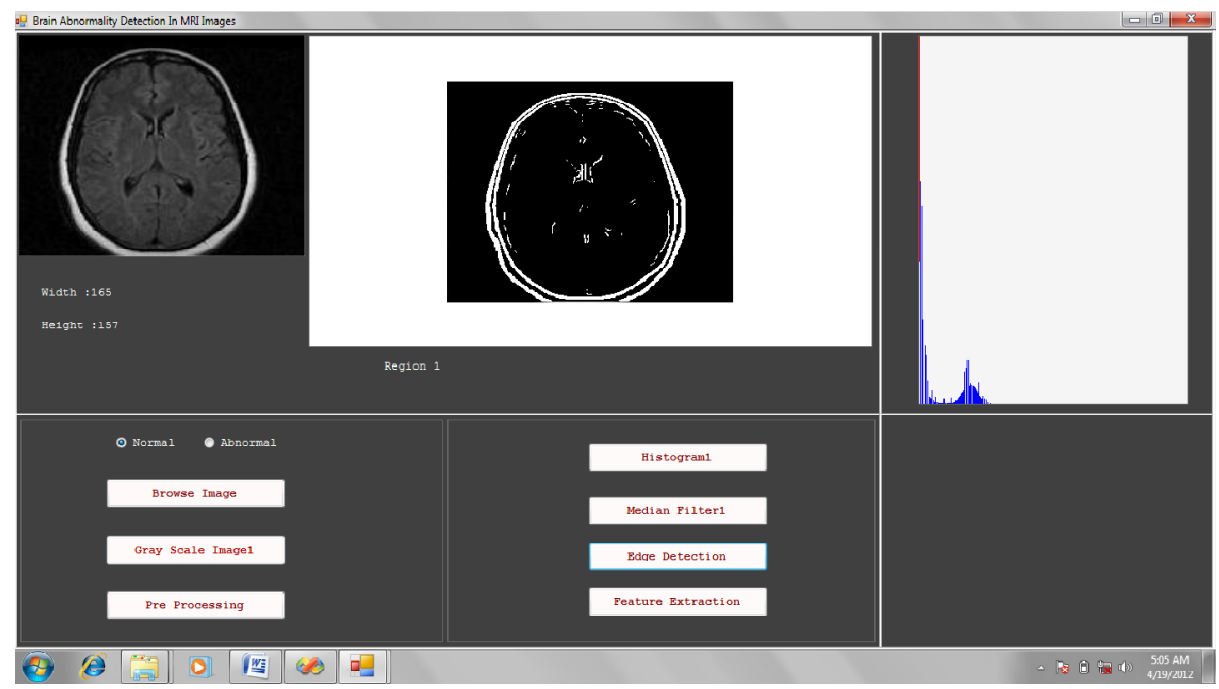

Fig 5. GUI of Edge detection of the selected MR image

\subsection{Feature Extraction}

After preprocessing the image, the morphological, textural, fractal, and/or intensity-based features for an image can be extracted. In order to extract the features, there are two different types of information available in the image one is the intensity values of the pixels and the other is their spatial interdependency. Although all feature extraction methods use the information on the intensity values, only a few use the spatial dependency between them. Employing only the intensity values, however, results in more sensitivity to the noise that arises from the stain artifacts and the image acquiring conditions. 
In this paper the intensity based features such as Skewness, Kurtosis, Smoothness Uniformity, Mean, and standard Deviation and Texture based features like Contrast, Entropy, Energy, correlation, homogeneity are extracted.

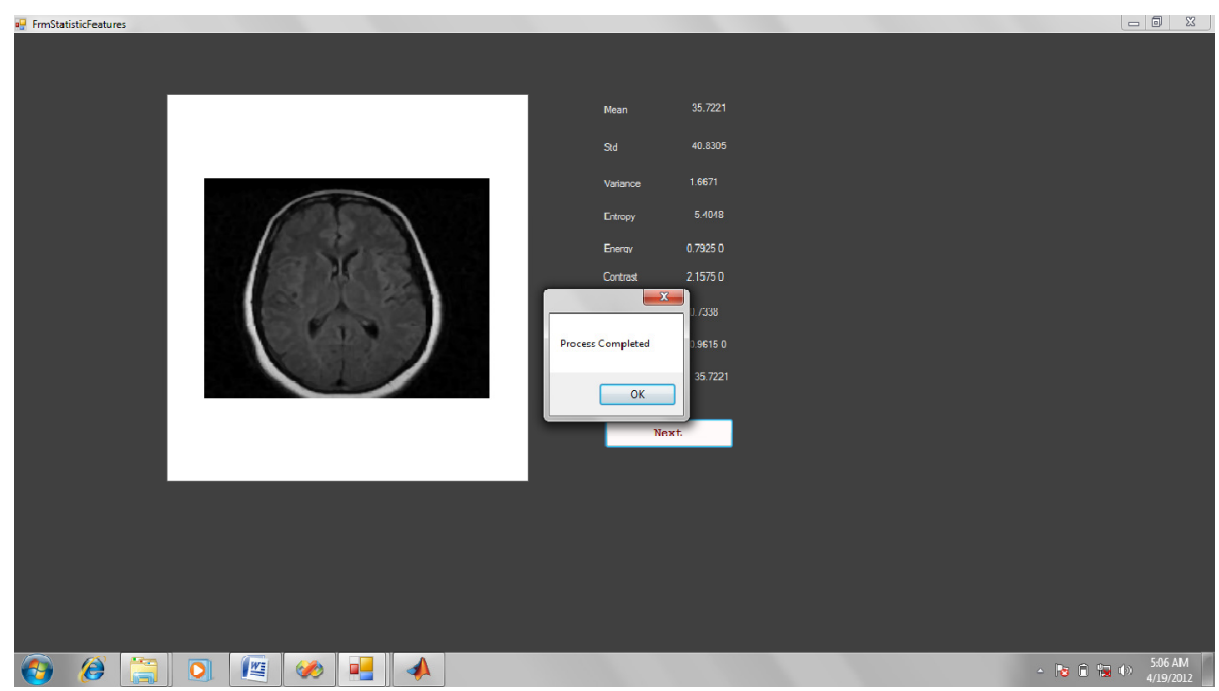

Fig 6. GUI of Feature extraction of the selected MR image

\subsection{ANN Training and Testing}

Neural Networks is a massively parallel distributed processor that has propensity for storing experimental knowledge in terms of synaptic weights and deals with more precise decisions.

The extracted features are fed as the input to the feed forward neural network, trained with supervised Error Back Propagation algorithm continuously for maximum samples till the error becomes minimum.

The structure of the neural network presented in this paper consists of an Input layer with 9 Neurons, two hidden layers, one with 5 neurons and second with 3 neurons, and one neuron at the output layer that distinguishes the image as normal and abnormal. 


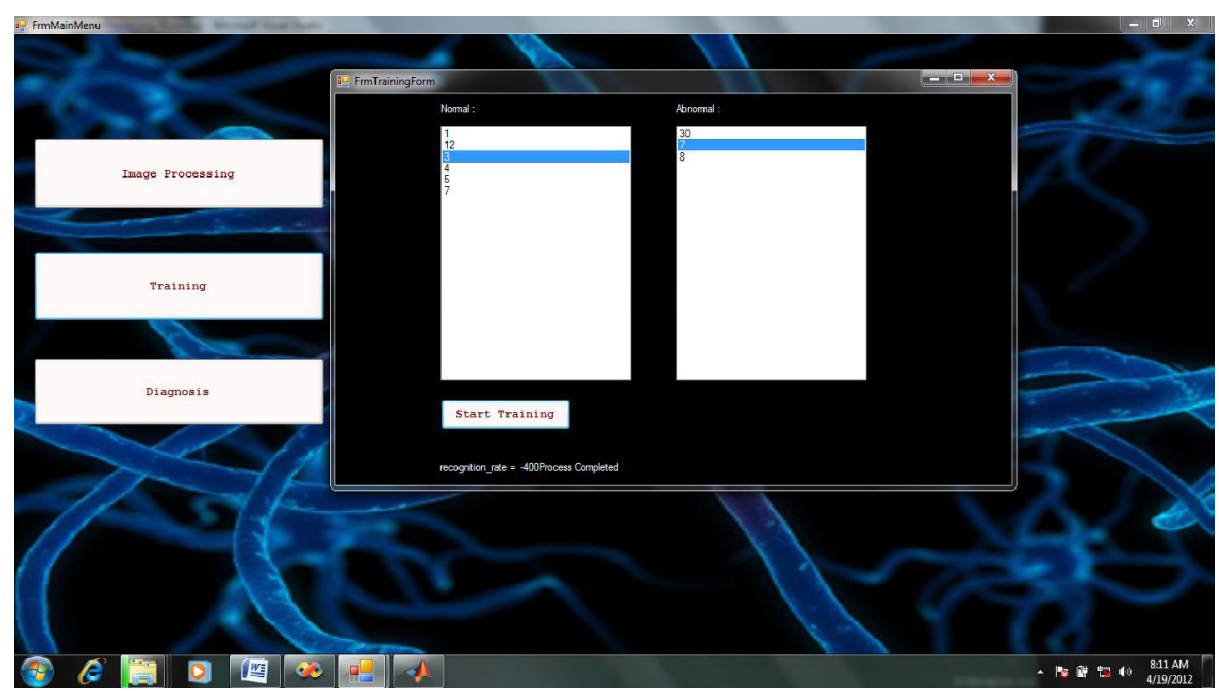

Fig 7. GUI of ANN Training

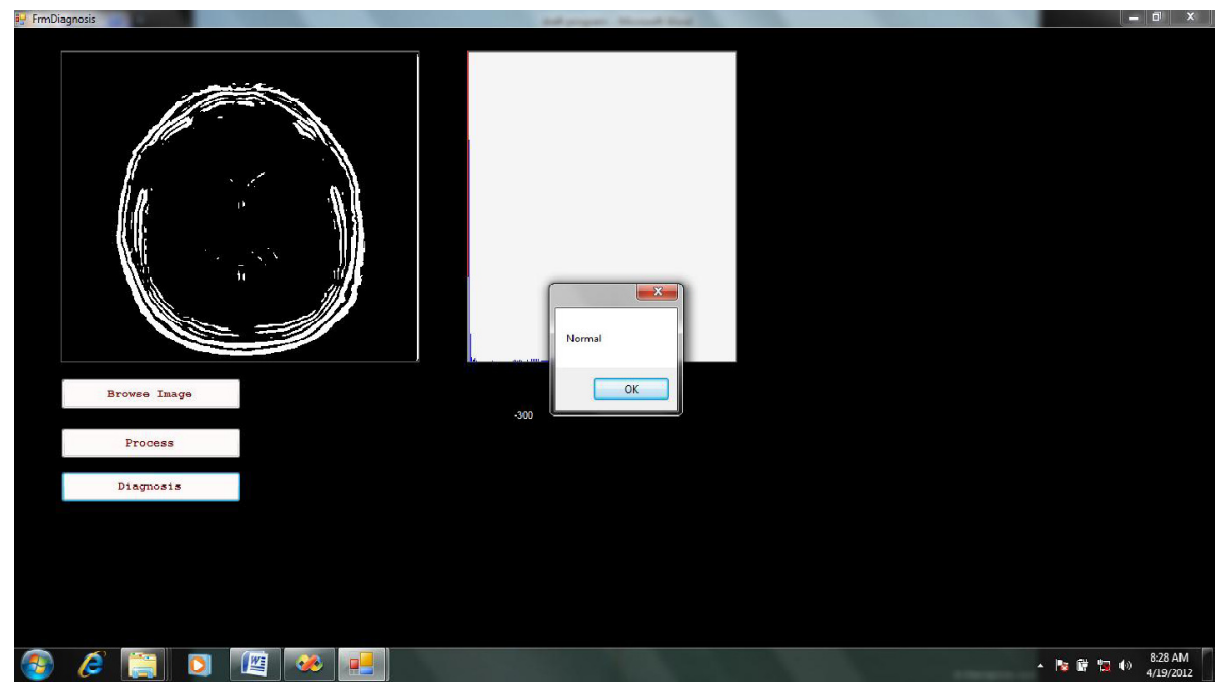

Fig 8. GUI of the diagnosis process

\section{RESULT AND ANALYSIS}

The following graph indicates the texture based feature values for normal (Benign) and abnormal (malignant) brain tumor images. 


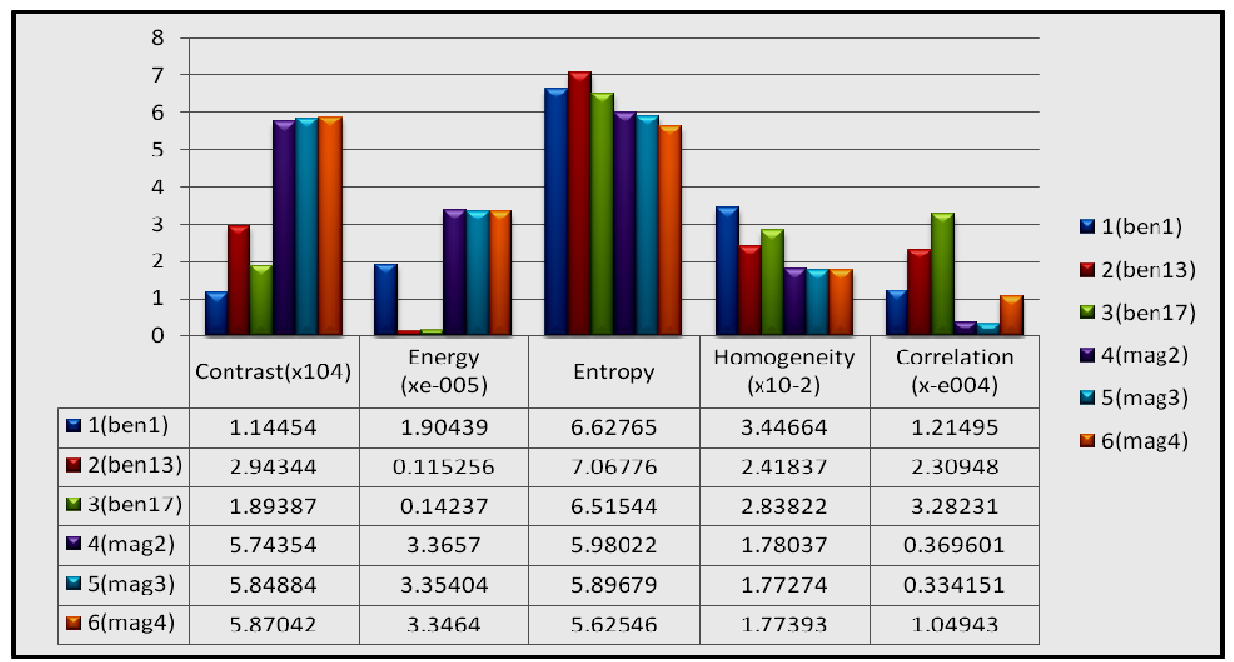

Fig 9. Graph indicating texture based feature values for normal and abnormal brain tumor images.

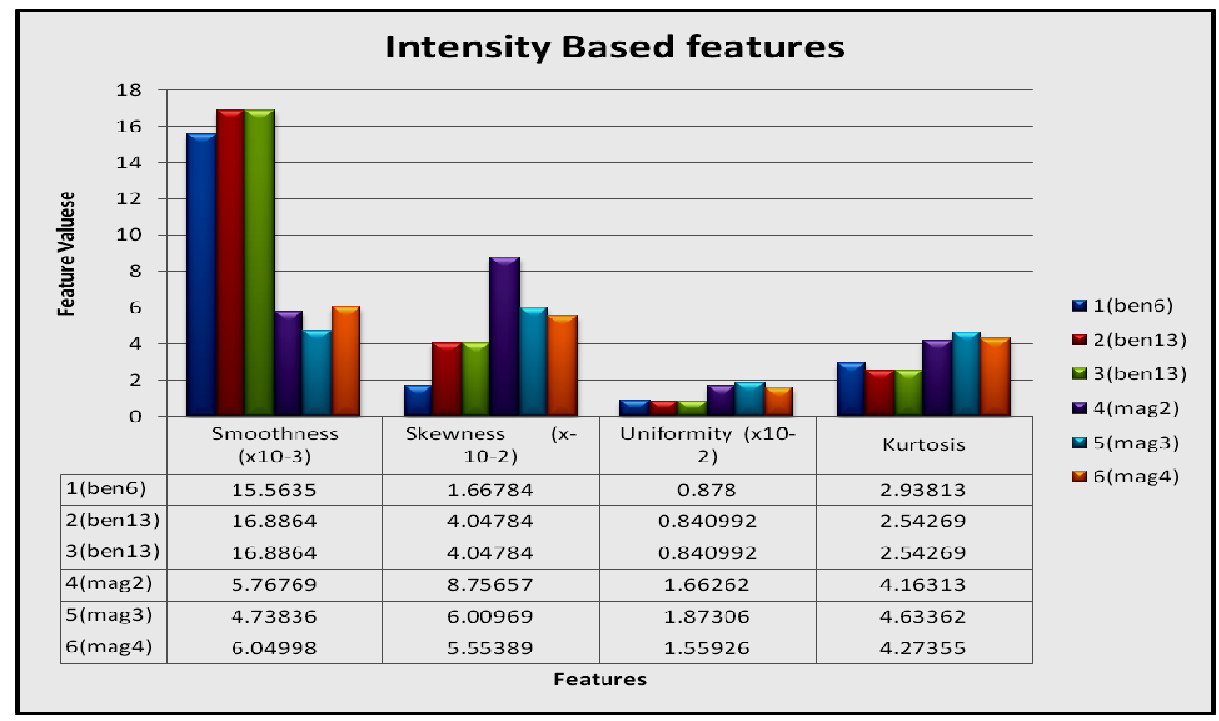

Fig 10. Graph indicating texture based feature values for normal and abnormal brain tumor images.

\section{CONCLUSION}

The presented method in this paper provides an alternative method to traditional human-based techniques, and optimally predicts the presence or absence of abnormality by using a noninvasive methodology. Processing of sample MR images of tumor and normal tissues enables the exploration and analysis by automatic means. The results obtained are accurate, fast and cost effective. The overall success rate of the system is found to be $92 \%$ when the network is tested with different number of training samples. The system is also validated using various validation techniques. 


\section{REFERENCES}

[1] Ashby LS, Troester MM, Shapiro WR "Central Nervous System Tumors" Cancer Therapeutics, Vol.1, pp. 475-513, 2006.

[2] Doolittle ND, "State of The Science in Brain Tumor Classification", Semin Oncol Nurs, vol.20, pp.224- 230, 2004.

[3] R. A. Lerski, K. Straughan, L. R. Schad, D.Boyce, S. Bluml, and I. Zuna, "MR ImageTexture Analysis- An approach To Tissue Characterization Magnetic Resonance Imaging, Vol. 11, pp. 873$887,1993$.

[4] H. Selvaraj1, S. Thamarai Selvi2, D. Selvathi3, L. Gewali1, “ Brain MRI Slices Classification Using Least Squares Support Vector Machine ”IC-MED, Vol.1, No. 1, Issue 1, Page 21 of $33,2007$.

[5] Jiayin Zhou, Vincent Chong,Tuan-Kay Lim, Jing Huang, "MRI Tumor Segmentation for Nasopharyngeal Carcinoma Using Knowledge-based Fuzzy Clustering" International Journal of Information Technology Vol. 8, No. 2 ,September 2002.

[6] Jason J. Corso, Member, IEEE, Eitan Sharon, Shishir Dube, Suzie El-Saden, Usha Sinha, and Alan Yuille, "Efficient Multilevel Brain Tumor Segmentation with Integrated Bayesian Model Classification" IEEE Transactions on Medical Imaging,pp.1-7 ,2007.

[7] Nathan Moon, 2Elizabeth Bullitt, 4Koen van Leemput, and 1;3Guido Gerig, " Automatic Brain and Tumor Segmentation”, MICCAI2002,LNCS2488(I) pp. 372-379,2002.

[8] Rajeev Ratan A, Sanjay Sharma B, S. K. SharmaC, "Brain Tumor Detection Based on MultiParameter MRI Image Analysis", ICGST-GVIP Journal, ISSN 1687-398X, Vol.9,Issue (III), June 2009. 